\title{
Heerfordt's Syndrome Associated with Trigeminal Nerve Palsy and Reversed Halo Sign
}

\author{
Go Makimoto ${ }^{1}$, Keita Kawakado ${ }^{1}$, Masamoto Nakanishi ${ }^{1}$, Tomoki Tamura ${ }^{1}$, Minori Noda ${ }^{2}$, \\ Satoko Makimoto ${ }^{3}$, Yumiko Sato ${ }^{4}$ and Shoichi Kuyama ${ }^{1}$
}

\begin{abstract}
:
Heerfordt's syndrome is a rare subtype of sarcoidosis and features a combination of facial palsy, parotid swelling, and uveitis, associated with a low-grade fever. Cases with two of three symptoms are called "incomplete Heerfordt's syndrome." Heerfordt's syndrome involving other cranial nerve symptoms is relatively rare. We herein report a case of incomplete Heerfordt's syndrome presenting with trigeminal nerve palsy and a reversed halo sign, a rare manifestation of pulmonary sarcoidosis. The histological diagnosis following a biopsy of the parotid gland and endobronchial ultrasound-guided trans-bronchial needle aspiration of the mediastinal lymph nodes was sarcoidosis. The symptoms and lung lesions improved after corticosteroid therapy.
\end{abstract}

Key words: Heerfordt's syndrome, trigeminal nerve palsy, reversed halo sign, endobronchial ultrasoundguided transbronchial needle aspiration

(Intern Med 60: 1747-1752, 2021)

(DOI: 10.2169/internalmedicine.6176-20)

\section{Introduction}

Heerfordt's syndrome is a subtype of sarcoidosis and occurs in approximately $1.6 \%$ of all sarcoidosis cases $(1,2)$. It is characterized as a combination of facial palsy, parotid gland enlargement, uveitis, and is associated with a lowgrade fever (3). Cases that manifest all three symptoms are called "complete Heerfordt's syndrome" (4). If only two of three characteristic symptoms are present, it is called "incomplete Heerfordt's syndrome." Heerfordt's syndrome associated with cranial nerve symptoms other than the facial nerve is relatively rare (5).

The reversed halo sign (RHS) is defined as a rounded focal area of ground-glass opacity surrounded by a complete or nearly complete ring of consolidation on high-resolution chest computed tomography (HRCT) (6). The RHS was initially recognized as a characteristic of cryptogenic organizing pneumonia (COP) $(7,8)$. However, the RHS was also reported in association with various diseases including infectious diseases; such as invasive fungal pneumonia, pneumo- cystis jirovecii pneumonia, tuberculosis, non-infectious diseases (e.g., granulomatosis with polyangiitis), pulmonary sarcoidosis, and neoplastic diseases (e.g., lymphomatoid granulomatosis and lung adenocarcinoma) (9). Lung field abnormality comprises approximately $40-50 \%$ of sarcoidosis cases (10), showing various patterns of shadows, such as multiple granular shadows with a perivascular and peripleural distribution associated with irregular thickening of the bronchovascular bundles (11). Sarcoidosis with RHS is a rare manifestation.

We herein report a unique case of incomplete Heerfordt's syndrome with trigeminal nerve palsy and an RHS. Following corticosteroid treatment, the symptoms and lung lesions (including the RHS) improved. We also reviewed the 20002020 literature in the MEDLINE database to search for cases of Heerfordt's syndrome and its clinical presentation.

\section{Case Report}

A 62-year-old woman was referred to our hospital to evaluate bilateral hilar lymphadenopathy (BHL) and multiple

\footnotetext{
${ }^{1}$ Department of Respiratory Medicine, National Hospital Organization Iwakuni Clinical Center, Japan, ${ }^{2}$ Department of Otorhinolaryngology, National Hospital Organization Iwakuni Clinical Center, Japan, ${ }^{3}$ Department of Radiology, National Hospital Organization Iwakuni Clinical Center, Japan and ${ }^{4}$ Department of Pathology, National Hospital Organization Iwakuni Clinical Center, Japan Received: August 25, 2020; Accepted: November 11, 2020; Advance Publication by J-STAGE: December 29, 2020 Correspondence to Dr. Go Makimoto, s_wolfjp@yahoo.co.jp
} 

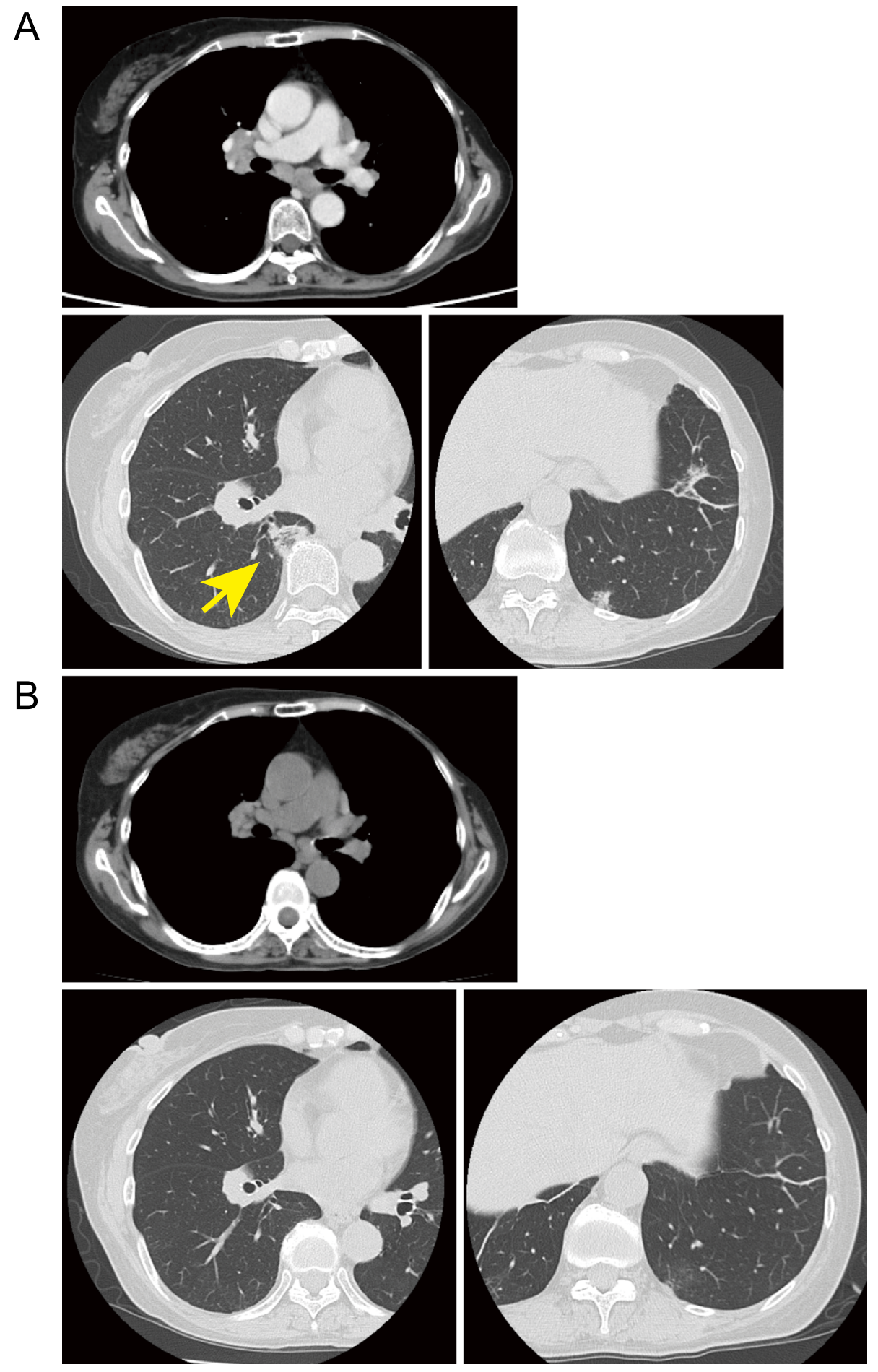

Figure 1. The computed tomography image before and after corticosteroid therapy (A: before corticosteroid therapy, B: two months after corticosteroid therapy). The reversed halo sign is indicated with a yellow arrow.

lung consolidations. She had a history of glaucoma and left mammectomy for breast cancer eight years earlier, and her family history was unremarkable. She was a never-smoker and a never-drinker. She had a 1.5-month history of dry cough and dyspnea, a 1-month history of dry mouth, and dry eye. One week later, she experienced blurred vision, conjunctiva injection, and pain of the left eye. She had been diagnosed with uveitis and treated at another hospital with antibiotics and a corticosteroid ophthalmic solution. After five days of treatment, she developed the same symptoms in her right eye, and chest radiography showed BHL, so she was referred to our hospital for the further diagnosis and treatment.

On a physical examination, she had uveitis and bilateral swollen parotid glands with a low-grade fever. Although she did not have facial nerve palsy, she had left-side maxillary nerve hyposensitivity caused by left trigeminal nerve palsy. Examinations of her heart and lungs were normal, as were 
her other cranial nerve and motor and sensory nerve examinations. Her serum lysozyme $(12.3 \mu \mathrm{g} / \mathrm{mL}$; normal range: 5.0-10.2 $\mu \mathrm{g} / \mathrm{mL}$ ) and soluble interleukin-2 (IL-2) receptor (1,701 IU/mL; normal range: 122-496 IU/mL) levels were elevated. Her serum angiotensin-converting enzyme, serum

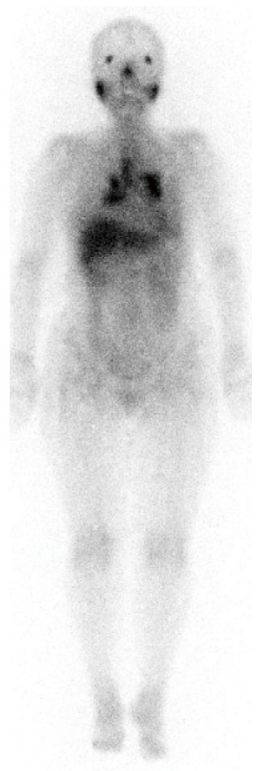

Figure 2. Gallium-67 scan before corticosteroid therapy showed an increased uptake in the lacrimal and parotid glands characterized by the "panda sign" and in the upper mediastinum and both hilar lymph nodes characterized by the "lambda sign". antinuclear antibody, anti-SS-A/B antibody, serum and urinary calcium levels, and serum D-dimer levels were all within normal limits. Her spirogram showed a normal pat-

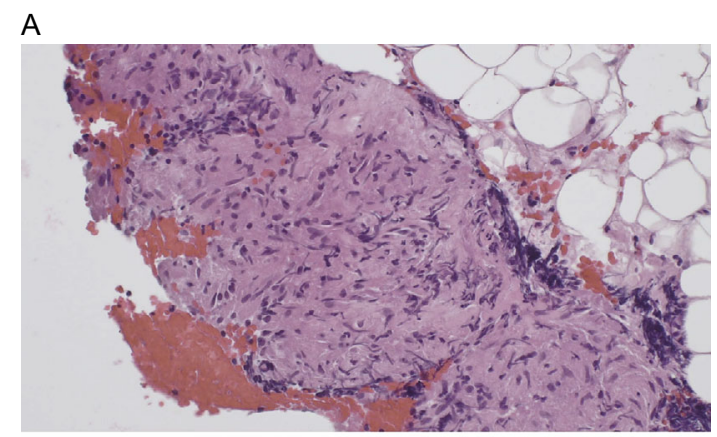

B

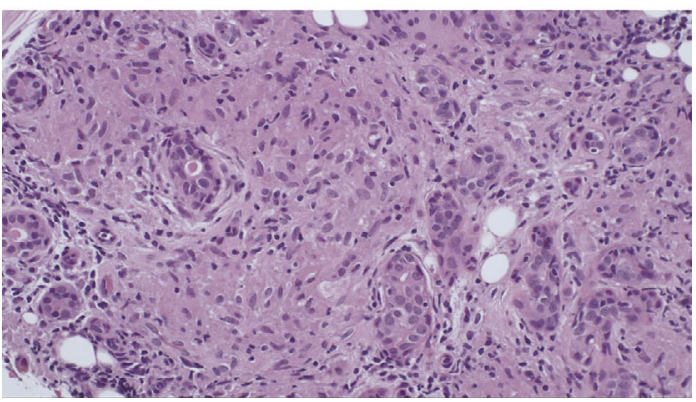

Figure 3. Endobronchial ultrasound-guided transbronchial needle aspiration for the mediastinal lymph node (A) and a needle biopsy of the parotid gland $(B)$ both revealed non-suppurative granulomatous lesions (Hematoxylin and Eosin staining, $\times 200)$.

Table 1-1. Literature Review of the Heerfordt's Syndrome Cases and Their Clinical Findings.

\begin{tabular}{|c|c|c|c|c|c|c|c|c|c|c|}
\hline No. & Year & Reference & Country & Age & Sex & $\begin{array}{l}\text { Clinical } \\
\text { type }\end{array}$ & $\begin{array}{l}\text { Facial } \\
\text { palsy }\end{array}$ & Uveitis & $\begin{array}{l}\text { Parotid } \\
\text { swelling }\end{array}$ & Fever \\
\hline 1 & 2002 & 12 & Italy & 54 & $\mathrm{~F}$ & Incomplete & - & + & $\mathrm{BL}$ & + \\
\hline 2 & 2002 & 13 & Germany & 33 & M & Complete & + & + & $\mathrm{BL}$ & + \\
\hline 3 & 2005 & 14 & USA & 44 & M & Incomplete & - & + & $\mathrm{BL}$ & + \\
\hline 4 & 2005 & 15 & Japan & 65 & $\mathrm{~F}$ & Incomplete & Right & - & $\mathrm{BL}$ & ND \\
\hline 5 & 2007 & 16 & Estonia & 22 & $\mathrm{~F}$ & Incomplete & Right & - & $\mathrm{BL}$ & - \\
\hline 6 & 2007 & 17 & Japan & 51 & $\mathrm{~F}$ & Incomplete & Left & + & ND & ND \\
\hline 7 & 2008 & 18 & Switzerland & 29 & M & Complete & Left & + & $\mathrm{BL}$ & + \\
\hline 8 & 2010 & 19 & USA & 39 & $\mathrm{~F}$ & Complete & Left & + & $\mathrm{BL}$ & + \\
\hline 9 & 2010 & 20 & Japan & 34 & M & Incomplete & - & + & $\mathrm{BL}$ & + \\
\hline 10 & 2013 & 21 & USA & 59 & $\mathrm{~F}$ & Complete & Left & + & Left & + \\
\hline 11 & 2013 & 22 & USA & 32 & $\mathrm{~F}$ & Incomplete & Right & - & $\mathrm{BL}$ & - \\
\hline 12 & 2015 & 23 & India & 52 & $\mathrm{~F}$ & Complete & BL & + & Left & + \\
\hline 13 & 2015 & 24 & Japan & 53 & M & Incomplete & Right & - & Right & ND \\
\hline 14 & 2015 & 24 & Japan & 55 & $\mathrm{~F}$ & Complete & Left & + & BL & ND \\
\hline 15 & 2016 & 25 & Japan & 34 & $\mathrm{~F}$ & Complete & Right & + & $\mathrm{BL}$ & ++ \\
\hline 16 & 2016 & 26 & India & 32 & M & Complete & $\mathrm{BL}$ & + & $\mathrm{BL}$ & + \\
\hline 17 & 2017 & 27 & Italy & 60 & $\mathrm{~F}$ & Complete & $\mathrm{BL}$ & + & $\mathrm{BL}$ & + \\
\hline 18 & 2017 & 28 & Brazil & 36 & $\mathrm{~F}$ & Incomplete & Left & + & ND & ND \\
\hline 19 & 2018 & 29 & India & 28 & M & Incomplete & - & + & $\mathrm{BL}$ & + \\
\hline 20 & 2019 & 30 & USA & 54 & $\mathrm{~F}$ & Incomplete & - & + & $\mathrm{BL}$ & + \\
\hline 21 & 2020 & Current case & Japan & 62 & $\mathrm{~F}$ & Incomplete & - & + & $\mathrm{BL}$ & + \\
\hline
\end{tabular}

F: female, M: male, BL: bilateral, ND: not described, Fever+: low-grade fever,++: high fever 
Table 1-2. Literature Review of the Heerfordt's Syndrome Cases and Their Clinical Findings (Continued).

\begin{tabular}{|c|c|c|c|c|c|c|c|}
\hline No. & Other cranial nerve symptoms & BHL & Lung lesion & RHS & $\begin{array}{l}\text { Cytology } \\
\text { (NCG site) }\end{array}$ & Therapy & Outcome \\
\hline 1 & - & + & - & - & Lip & PSL 50mg & Partially improved \\
\hline 2 & V2 (dysesthesia) & + & - & - & Parotid & ND & ND \\
\hline 3 & - & + & - & - & Parotid & PSL 60mg & Completely improved \\
\hline 4 & - & + & - & - & $\begin{array}{l}\text { Subcutaneous } \\
\text { mass }\end{array}$ & PSL 50mg & Completely improved \\
\hline 5 & - & + & - & - & Lip & steroid & Completely improved \\
\hline 6 & - & - & $\begin{array}{l}\text { Small nodular } \\
\text { lesions }\end{array}$ & - & TBLB & ND & $\mathrm{ND}$ \\
\hline 7 & V1-V2 (hyposensitivity) & + & - & - & $\begin{array}{l}\text { Transbronchial } \\
\text { lymph node biopsy }\end{array}$ & $\mathrm{PSL}+\mathrm{AZA}$ & Completely improved \\
\hline 8 & - & + & - & - & TBLB & steroid & Completely improved \\
\hline 9 & - & + & - & - & Skin & PSL $15 \mathrm{mg}$ & Not improved \\
\hline 10 & - & + & $\begin{array}{l}\text { bibasilar interstitial } \\
\text { lung disease }\end{array}$ & - & Pre-auricular LN & PSL & Completely improved \\
\hline 11 & - & - & - & - & Parotid & PSL 60mg & Completely improved \\
\hline 12 & - & + & - & - & Parotid & PSL & Partially improved \\
\hline 13 & - & - & - & - & Eyelid, thigh LN & PSL 40mg & Completely improved \\
\hline 14 & - & + & - & - & - & PSL 60mg & Partially improved \\
\hline 15 & - & + & - & - & $\begin{array}{c}\text { Supraclavicular } \\
\text { LN, EBUS-TBNA }\end{array}$ & PSL 30mg & Completely improved \\
\hline 16 & - & + & - & - & Parotid & steroid & Completely improved \\
\hline 17 & $\begin{array}{l}\text { VI (eye movement limitation), } \\
\text { XII (deviation of the tongue) }\end{array}$ & - & consolidation & - & Parotid & PSL 60mg & Partially improved \\
\hline 18 & - & - & $\begin{array}{l}\text { small parenchymal } \\
\text { nodules }\end{array}$ & - & Skin & PSL+MTX & Completely improved \\
\hline 19 & $\begin{array}{l}\text { III (drooping of the eyelid), } \\
\text { X (difficulty in swallowing) }\end{array}$ & + & $\begin{array}{l}\text { multiple lung } \\
\text { nodules }\end{array}$ & - & Cervical LN & PSL & Completely improved \\
\hline 20 & - & + & $\begin{array}{c}\text { Interstitial } \\
\text { opacities }\end{array}$ & - & Skin & MTX 10mg & ND \\
\hline 21 & V2 (hyposensitivity) & + & $\begin{array}{l}\text { multiple patchy } \\
\text { shadows }\end{array}$ & + & $\begin{array}{c}\text { Parotid, } \\
\text { EBUS-TBNA }\end{array}$ & PSL 30mg & Partially improved \\
\hline
\end{tabular}

BHL: bilateral hilar lymphadenopathy, RHS: reversed halo sign, NCG: non-caseating epithelioid granuloma, TBLB: transbronchial lung biopsy, LN: lymph node, EBUS-TBNA: endobronchial ultrasound-guided transbronchial needle aspiration, ND: not described, PSL: prednisolone, AZA: azathioprine, MTX: methotrexate

tern (VC 2.79 L, \%VC 106.0\%, FEV1 2.41 L, FEV1\% $85.2 \%)$.

Chest computed tomography (CT) revealed bilateral parotid gland swelling, hilar and mediastinal lymphadenopathy, and bilateral multiple peripheral consolidations, including the RHS (Fig. 1A). A gallium-67 scan also showed bilateral symmetric areas of an increased uptake in the lacrimal and parotid gland (characterized as panda sign) as well as in the upper mediastinum and both hila (characterized as lambda sign) (Fig. 2).

Two days after she was referred to our hospital, her mediastinal lymph node was biopsied via endobronchial ultrasound-guided transbronchial needle aspiration (EBUSTBNA). The biopsy results revealed non-necrotizing granulomatous lymphadenitis (Fig. 3A). Her swollen parotid gland was also biopsied, revealing a non- necrotizing granulomatous lesion (Fig. 3B). Samples of bronchoalveolar lavage fluid showed an elevated lymphocyte proportion (66.7\%) and CD4/CD8 ratio (9.04), which supported the diagnosis of pulmonary sarcoidosis.

Based on these findings, we diagnosed her with incom- plete Heerfordt's syndrome, and the patient was treated with corticosteroid therapy (oral prednisolone, $30 \mathrm{mg}$ per day). Within a few weeks, her blurred vision, dry mouth, dry eye, dry cough, and dyspnea gradually improved. Her left-side maxillary nerve hyposensitivity caused by left trigeminal nerve palsy also improved. She did not show any facial nerve palsy in the clinical course. The serum soluble IL-2 receptor and lysozyme levels decreased to $622 \mathrm{IU} / \mathrm{mL}$ and $4.7 \mu \mathrm{g} / \mathrm{mL}$, respectively, on day 30. Chest CT also showed improvement in the BHL and RHS (Fig. 1B). The prednisolone dose was reduced to $15 \mathrm{mg}$ for 3 months without recurrence of any symptoms.

\section{Discussion}

Heerfordt's syndrome was first described by the Danish ophthalmologist Christian Fredrick Heerfordt in 1909 (3). This syndrome combines uveitis, parotid gland swelling, and facial nerve palsy in association with a low-grade fever. Later, this syndrome was found to be associated with sarcoidosis (4). Cases that present with all three symptoms and a 
fever are called "complete Heerfordt's syndrome," and cases with two symptoms are called "incomplete Heerfordt's syndrome." Darlington et al. reviewed the characteristics of sarcoidosis and reported that, of 1,000 patients with sarcoidosis, only $3(0.3 \%)$ had complete Heerfordt's syndrome, and $13(1.3 \%)$ had incomplete Heerfordt's syndrome (2).

To analyze the various characteristics of Heerfordt's syndrome, we searched for the MEDLINE database for literature published between 2000 and 2020 using the term "Heerfordt's syndrome," which yielded 40 articles. After excluding articles not written in English or not specifically related to Heerfordt's syndrome, we reviewed 19 articles concerning 21 cases, including our own case (Table) (12-30). The median patient age was 44 (range 22-65) years old, and there were 7 men and 14 women. Among the 21 cases, 9 were considered complete Heerfordt's syndrome. Regarding cranial nerve symptoms other than the facial nerve, five cases showed cranial nerve symptoms, including three cases with trigeminal nerve symptoms. Although the majority of cases showed BHL (16/21), only 7 showed lung lesions. The lung lesion patterns were varied. We identified three cases with nodular patterns, two with interstitial patterns, one with a consolidation pattern, and one (our own) with a patchy pattern. As for the RHS, there have been no Heerfordt's syndrome cases associated with the RHS aside from the present case.

Marchiori et al. reviewed 79 RHS cases and reported that granulomatous diseases, such as pulmonary tuberculosis or sarcoidosis, tended to have small nodules in the wall of the RHS compared with other diseases manifesting RHS, such as cryptogenic organizing pneumonia (31). In the present case, the RHS was relatively small, so there were no significant nodules inside the RHS. There have been several sarcoidosis cases diagnosed by a transbronchial lung biopsy with the RHS (11, 32-34). In those reports, Fujii et al. noted that biopsy specimen of the ring in the late phase of the RHS showed no significant granulomatous lesions, and the RHS showed a time-phase heterogeneity (32). In our case, we biopsied not only the mediastinal lymph node but also the "within lesion" of the RHS via endobronchial ultrasonography using the guide sheath method. There was no significant granulomatous lesion. In such cases, EBUSTBNA for the mediastinal lymph node may be more effective for diagnosing sarcoidosis pathologically.

The standard treatment strategy for Heerfordt's syndrome has not been established because of the rarity of this syndrome. However, corticosteroid treatment based on the neurosarcoidosis should be applied for cases in which facial nerve palsy is frequently observed (24). The majority of Heerfordt's syndrome cases, including our own, have responded to corticosteroid therapy (Table1-2).

We encountered a rare case of incomplete Heerfordt's syndrome associated with trigeminal nerve palsy and the RHS. When the RHS is observed with parotid swelling or uveitis, we should consider Heerfordt's syndrome and pathologically differentiate the syndrome from other lesions using techniques such as EBUS-TBNA.

The authors state that they have no Conflict of Interest (COI).

\section{References}

1. Greenberg G, Anderson R, Sharpstone P, James DG. Enlargement of parotid gland due to sarcoidosis. Br Med J 2: 861-862, 1964.

2. Darlington P, Tallstedt L, Padyukov L, et al. HLA-DRB1* alleles and symptoms associated with Heerfordt's syndrome in sarcoidosis. Eur Respir J 38: 1151-1157, 2011.

3. Heerfordt C. [About a "Febris uveoparotidea subchronica" located on the parotid gland and the uvea of the eye and often complicated with paresis cerebrospinal nerves]. Arch Ophthalmol 70: 254-273, 1909 (in German).

4. Bruins-Slot WJ, Foedblod J, Goslings J. [Besnier-Boeck (Schaumann) disease and uveo parotitis (Heerfordt)]. Acta Med Scand 94: 74-97, 1938 (in German).

5. Otani K, Noda K, Ukichi T, Kingetsu I, Kurosaka D. A case of abortive type of Heerfordt syndrome associated with paralysis of trigeminal nerve. Nihon Rinsho Meneki Gakkai Kaishi 36: 115121, 2013 (in Japanese, Abstraet in English).

6. Hansell DM, Bankier AA, MacMahon H, McLoud TC, Müller NL, Remy J. Fleischner Society: glossary of terms for thoracic imaging. Radiology 246: 697-722, 2008.

7. Voloudaki AE, Bouros DE, Froudarakis ME, Datseris GE, Apostolaki EG, Gourtsoyiannis NC. Crescentic and ring-shaped opacities. CT features in two cases of bronchiolitis obliterans organizing pneumonia (BOOP). Acta Radiol 37: 889-892, 1996.

8. Kim SJ, Lee KS, Ryu YH, et al. Reversed halo sign on highresolution CT of cryptogenic organizing pneumonia: diagnostic implications. AJR Am J Roentgenol 180: 1251-1254, 2003.

9. Godoy MC, Viswanathan C, Marchiori E, et al. The reversed halo sign: update and differential diagnosis. Br J Radiol 85: 1226-1235, 2012.

10. Morimoto T, Azuma A, Abe S, et al. Epidemiology of sarcoidosis in Japan. Eur Respir J 31: 372-379, 2008.

11. Kumazoe H, Matsunaga K, Nagata N, et al. "Reversed halo sign" of high-resolution computed tomography in pulmonary sarcoidosis. J Thorac Imaging 24: 66-68, 2009.

12. Di Stefano F, Paganelli R, Verna N, Di Gioacchino M. Cardiac sarcoidosis presenting as Heerfordt's syndrome. Am J Med 112: 594-595, 2002.

13. Fischer T, Filimonow S, Petersein J, Zimmer C, Beyersdorff D, Guski H. Diagnosis of Heerfordt's syndrome by state-of-the-art ultrasound in combination with parotid biopsy: a case report. Eur Radiol 12: 134-137, 2002.

14. Blair MP, Rizen M. Heerfordt syndrome with internal ophthalmoplegia. Arch Ophthalmol 123: 1017, 2005.

15. Sugawara Y, Sakayama K, Sada E, et al. Heerfordt syndrome initially presenting with subcutaneous mass lesions: usefulness of gallium-67 scans before and after treatment. Clin Nucl Med 30: 732-733, 2005.

16. Tamme T, Leibur E, Kulla A. Sarcoidosis (Heerfordt syndrome): a case report. Stomatologija 9: 61-64, 2007.

17. Ueda $N$, Satoh $T$, Yamamoto $T$, Yokozeki H. Nodular cystic fat necrosis in Heerfordt's syndrome. J Eur Acad Dermatol Venereol 21: 708-709, 2007.

18. Petropoulos IK, Zuber JP, Guex-Crosier Y. Heerfordt syndrome with unilateral facial nerve palsy: a rare presentation of sarcoidosis. Klin Monbl Augenheilkd 225: 453-456, 2008.

19. Evanchan J, Barreiro TJ, Gemmel D. Uveitis, salivary gland swelling, and facial nerve palsy in a febrile woman. Jaapa 23: 48-50, 2010.

20. Yagi T, Hattori H, Ohira M, et al. Progressive multifocal leukoen- 
cephalopathy developed in incomplete Heerfordt syndrome, a rare manifestation of sarcoidosis, without steroid therapy responding to cidofovir. Clin Neurol Neurosurg 112: 153-156, 2010.

21. Denny MC, Fotino AD. The Heerfordt-Waldenström syndrome as an initial presentation of sarcoidosis. Proc (Bayl Univ Med Cent) 26: 390-392, 2013.

22. Dua A, Manadan A. Images in clinical medicine. Heerfordt's syndrome, or uveoparotid fever. N Engl J Med 369: 458, 2013.

23. Chappity P, Kumar R, Sahoo AK. Heerfordt's syndrome presenting with recurrent facial nerve palsy: case report and 10-year literature review. Sultan Qaboos Univ Med J 15: e124-e128, 2015.

24. Fujiwara K, Furuta Y, Fukuda S. Two cases of Heerfordt's syndrome: a rare manifestation of sarcoidosis. Case Rep Otolaryngol 2016: 3642735, 2016.

25. Makimoto G, Miyahara N, Yoshikawa M, et al. Heerfordt's syndrome associated with a high fever and elevation of TNF- $\alpha$. Acta Med Okayama 70: 273-277, 2016.

26. Srirangaramasamy J, Kathirvelu S. A rare case of Heerfordt's syndrome with bilateral facial palsy. Indian J Otolaryngol Head Neck Surg 71: 1027-1029, 2016.

27. Broggi G, Reggio E, Giuliano L, Palmucci S, Caltabiano R, Lanzafame S. Parotid gland involvement in Heerfordt syndrome: a case report. Pathologica 109: 418-420, 2017.

28. Fraga RC, Kakizaki P, Valente NYS, Portocarrero LKL, Teixeira
MFS, Senise PF. Do you know this syndrome? HeerfordtWaldenström syndrome. An Bras Dermatol 92: 571-572, 2017.

29. Malkani R, Karia R. Leprosy or sarcoidosis? A diagnostic dilemma! J Family Med Prim Care 7: 1106-1108, 2018.

30. Ramachandran V, Haidari W, Ahn C, Tull R, Jorizzo JL. Uveoparotid fever as a presentation of sarcoidosis. Proc (Bayl Univ Med Cent) 32: 616-618, 2019.

31. Marchiori E, Zanetti G, Escuissato DL, et al. Reversed halo sign: high-resolution CT scan findings in 79 patients. Chest 141: 12601266, 2012.

32. Fujii M, Ida M, Enomoto N, et al. A case of sarcoidosis manifesting as annular opacities after multiple nodular shadows. Nihon Kokyuki Gakkai Zasshi 40: 970-974, 2002.

33. Marchiori E, Zanetti G, Mano CM, Hochhegger B, Irion KL. The reversed halo sign: another atypical manifestation of sarcoidosis. Korean J Radiol 11: 251-252, 2010.

34. Zhan X, Zhang L, Wang Z, Jin M, Liu M, Tong Z. Reversed halo sign: presents in different pulmonary diseases. PLoS One 10: e0128153, 2015.

The Internal Medicine is an Open Access journal distributed under the Creative Commons Attribution-NonCommercial-NoDerivatives 4.0 International License. To view the details of this license, please visit (https://creativecommons.org/licenses/ by-nc-nd/4.0/).

(C) 2021 The Japanese Society of Internal Medicine Intern Med 60: 1747-1752, 2021 